\title{
Wind Velocity Data Interpolation Using Rational Cubic Spline
}

\author{
Samsul Ariffin Bin Abdul Karim ${ }^{1, *}$ and S. Suresh Kumar Raju ${ }^{2}$ \\ ${ }^{1}$ Fundamental and Applied Sciences Department and Centre for Smart Grid Energy Research \\ (CSMER), Institute of Autonomous System, Universiti Teknologi PETRONAS, Bandar Seri \\ Iskandar, 32610 Seri Iskandar, Perak DR, Malaysia. \\ ${ }^{2}$ Department of Mathematics and Statistics, King Faisal University, Saudi Arabia.
}

\begin{abstract}
Wind velocity data is always having positive value and the minimum value approximately close to zero. The standard cubic spline interpolation (not-a-knot and natural) as well as cubic Hermite polynomial may be produces interpolating curve with negative values on some subintervals. To cater this problem, a new rational cubic spline with three parameters is constructed. This rational spline will be used to preserve the positivity of the wind velocity data. Numerical results shows that the proposed scheme work very well and give visually pleasing interpolating curve on the given domain.
\end{abstract}

\section{Introduction}

Meteorological data parameters are important especially when the scientist dealing with some problems such as dengue transmission at certain location, the amount of rainfall received in a month as well as the earthquake events or history etc. Some common meteorological data are rainfall, temperature (maximum, minimum and average), humidity, wind velocity or speed as well as wind direction. $\mathrm{Wu}$ et al. [8] studied the multiquadric interpolation to approximate the wind speed data. Their method is only capable to approximate the wind velocity where the interpolations to all data points are lost. Karim [4] discussed the monotonic interpolating cure by using rational cubic spline of Karim and Kong [3]. Sarfraz et al. [6] discussed the positivity preserving interpolation by using rational cubic spline with two parameters. The main drawback of [6] is that, on some intervals, the positivity of the data is not preserved as proved by Qin et al. [7]. Hussain et al. [2] scheme also does not guarantee to produces positive interpolating curve everywhere.

In this study, a new rational cubic spline in the form of cubic numerator and quadratic denominator is proposed. This rational spline has three parameters for shape modification. In order to produce positive rational interpolant, the sufficient condition for the positivity of the rational spline interpolant is derived on one parameter meanwhile the other two parameters can be used to refine the shape of positive interpolating curve.

\footnotetext{
*Corresponding author: samsul_ariffin@utp.edu.my
} 


\section{Rational cubic spline interpolant}

The construction of new rational cubic spline is discussed below. Given data points $\left\{\left(x_{i}, f_{i}\right), i=0,1, \ldots, n\right\}$ which satisfy $x_{0}<x_{1}<\cdots<x_{n}$ and the first derivatives $d_{i}, i=0,1, \ldots, n$ . Let the step size $h_{i}=x_{i+1}-x_{i}$, the gradient is $\Delta_{i}=\left(f_{i+1}-f_{i}\right) / h_{i}$ and $\theta=\left(x-x_{i}\right) / h_{i}$ where $0 \leq \theta \leq 1$. For each sub-intervals $x \in\left[x_{i}, x_{i+1}\right], i=0,1, \ldots, n-1$, the rational cubic spline with three parameters $\alpha_{i}, \beta_{i}$, and $\gamma_{i}$, for $i=0,1, \ldots, n-1$ is defined by

$$
S(x)=S_{i}(x)=\frac{P_{i}(\theta)}{Q_{i}(\theta)}
$$

where

$$
\begin{aligned}
& P_{i}(\theta)=(1-\theta)^{2} U_{i}+(1-\theta)^{2} \theta V_{i}+(1-\theta) \theta^{2} W_{i}+\theta^{2} Z_{i} \\
& Q_{i}(\theta)=\alpha_{i}(1-\theta)^{2}+2 \gamma_{i}(1-\theta) \theta+\beta_{i} \theta^{2}
\end{aligned}
$$

In this study, we construct $C^{1}$ rational cubic spline that satisfies the following conditions:

$$
\begin{aligned}
S\left(x_{i}\right) & =f_{i}, & S^{(1)}\left(x_{i}\right) & =d_{i} \\
S\left(x_{i+1}\right) & =f_{i+1}, & S^{(1)}\left(x_{i+1}\right) & =d_{i+1}
\end{aligned}
$$

By using (2) and the rational cubic spline in (1), the unknowns, $U_{i}, V_{i}, W_{i}$ and $Z_{i}$ for $i=0,1, \ldots, n-1$ are:

$$
\begin{aligned}
& U_{i}=\alpha_{i} f_{i}, V_{i}=2 \gamma_{i} f_{i}+\alpha_{i} h_{i} d_{i}, \\
& \mathrm{~W}_{i}=\gamma_{i} f_{i+1}-\beta_{i} h_{i} d_{i+1}, \mathrm{Z}_{i}=\beta_{i} f_{i+1}
\end{aligned}
$$

With this value, the rational cubic spline defined in (1) can be written as

$$
S(x)=\frac{(1-\theta)^{2} \alpha_{i} f_{i}+(1-\theta)^{2} \theta\left(2 \gamma_{i} f_{i}+\alpha_{i} h_{i} d_{i}\right)+(1-\theta) \theta^{2}\left(2 \gamma_{i} f_{i+1}-\beta_{i} h_{i} d_{i+1}\right)+\theta^{2} \beta_{i} f_{i+1}}{\alpha_{i}(1-\theta)^{2}+2 \gamma_{i}(1-\theta) \theta+\beta_{i} \theta^{2}}
$$

When $\alpha_{i}=\beta_{i}=\gamma_{i}=1$, the rational cubic spline in (3) is reduce to the cubic Ball polynomial.

\section{Positivity preserving data interpolation}

The rational cubic spline defined by (3) can be used to interpolate the wind velocity data (or any other positive data) by supplying any positive value to the shape parameters $\alpha_{i}, \beta_{i}$, and $\gamma_{i}$, for $i=0,1, \ldots, n-1$. Table 1 shows the wind velocity data obtained in $\mathrm{Wu}$ et al. [8]. Fig. 1 shows some examples of interpolating curve by varying $\alpha_{i}, \beta_{i}$, and $\gamma_{i}$.

Table 1. A wind data [8]

\begin{tabular}{|c|c|c|c|c|c|c|c|}
\hline$i$ & 0 & 1 & 2 & 3 & 4 & 5 & 6 \\
\hline$x_{i}$ & 0 & 0.25 & 0.5 & 1 & 1.2 & 1.8 & 2 \\
\hline$f_{i}$ & 2 & 0.8 & 0.5 & 0.1 & 1 & 0.5 & 1 \\
\hline
\end{tabular}




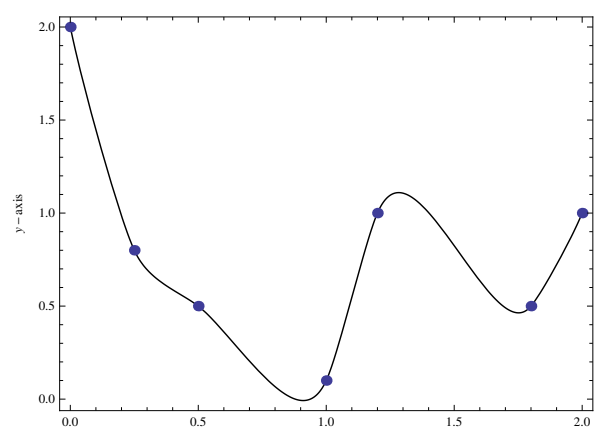

(a) $\alpha_{i}=\beta_{i}=1, \gamma_{i}=2$

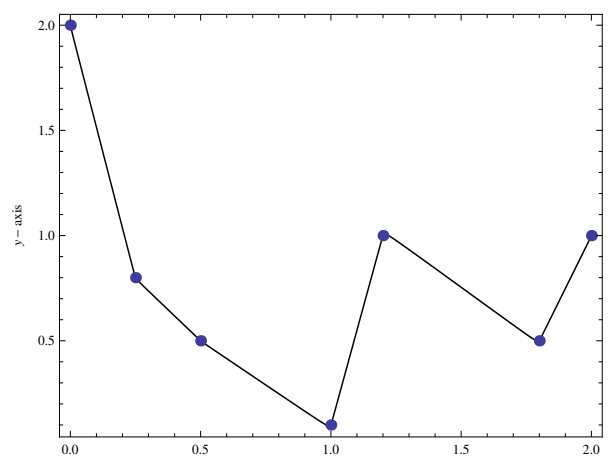

(c) $\alpha_{i}=\beta_{i}=1, \gamma_{i}=50$

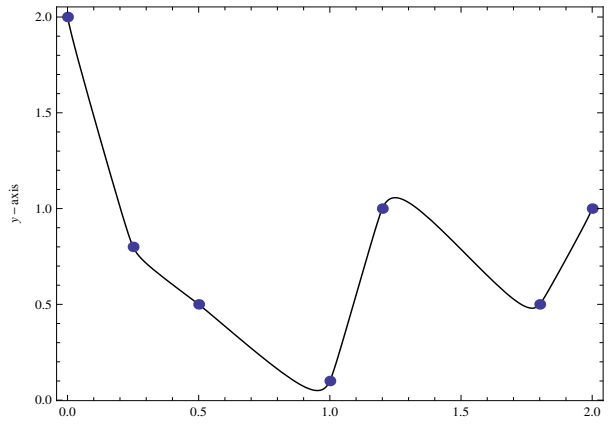

(b) $\alpha_{i}=\beta_{i}=1, \gamma_{i}=5$

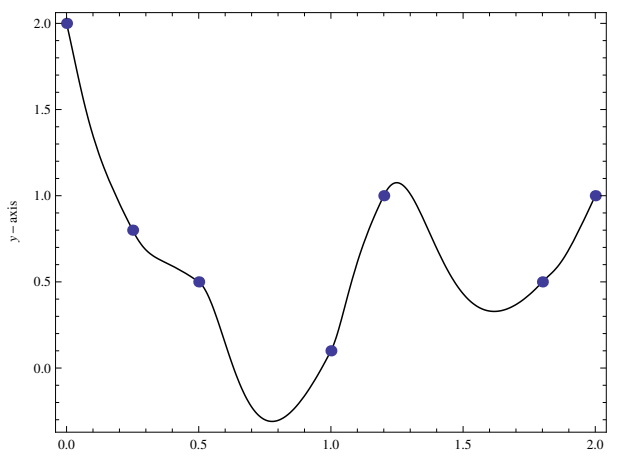

(d) $\alpha_{i}=\gamma_{i}=1, \beta_{i}=5$

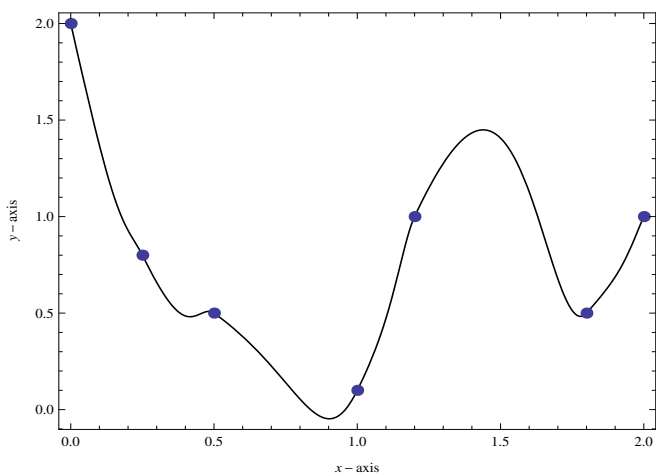

(e) $\alpha_{i}=5, \gamma_{i}=\beta_{i}=1$

Fig. 1. Various interpolating curves

It is not easy to find suitable value of each parameter that will ensure that the resulting interpolating curve is positive everywhere. The automated choice of parameter $\gamma_{i}>0$, $i=0,1, \ldots, n-1$ is derived that will ensure the positive rational interpolant is produced. Since we are using rational spline, then data must be strictly positive in order to avoid the dividing by zero in the mathematical derivation later:

$$
f_{i}>0, \quad i=0,1,2, \ldots, n
$$

Condition (4) is the same as 


$$
S_{i}(x)>0, \quad i=0,1,2, \ldots, n-1
$$

When $\alpha_{i}, \beta_{i}>0$, and $\gamma_{i} \geq 0$ for $i=0,1, \ldots, n-1$, the quadratic polynomial $Q_{i}(\theta)$ is always positive such that $Q_{i}(\theta)>0$. Thus $S_{i}(x)>0$, if and only if the cubic polynomial $P_{i}(\theta)$ [7] is positive i.e. $P_{i}(\theta)>0$ for all $i=0,1,2, \ldots, n-1$. Theorem 1 state the sufficient condition for the positivity of the rational cubic interpolant defined by (3).

Theorem 1. For strictly positive data, the rational cubic spline interpolant $S(x)$ in (3) will be positive everywhere if in each sub-interval $\left[x_{i}, x_{i+1}\right], i=0,1, \ldots, n-1$, the parameter $\gamma_{i}>0$ , $i=0,1, \ldots, n-1$ satisfies:

$$
\gamma_{i}>\operatorname{Max}\left\{0,-\alpha_{i}\left(\frac{h_{i} d_{i}}{2 f_{i}}+\frac{1}{2}\right), \beta_{i}\left(\frac{h_{i} d_{i+1}}{2 f_{i+1}}-1\right)-\frac{1}{2}\right\}, \quad \alpha_{i}, \beta_{i}>0
$$

\section{Proof.}

Firstly, the first derivative of $P_{i}^{\prime}(\theta)$ is equal to

$$
P_{i}^{\prime}(\theta)=\frac{-2 \alpha_{i} f_{i}(1-\theta)+V_{i}\left((1-\theta)^{2}-2 \theta(1-\theta)\right)+W_{i}\left(-\theta^{2}+2 \theta(1-\theta)\right)+2 \beta_{i} f_{i+1} \theta}{h_{i}}
$$

The sufficient conditions for positivity of the rational cubic spline are given below:

and

$$
P_{i}^{\prime}(0)>\frac{-3 P_{i}(0)}{h_{i}} \Rightarrow \frac{-2 \alpha_{i} f_{i}+2 \gamma_{i} f_{i}+\alpha_{i} h_{i} d_{i \mathrm{i}}}{h_{i}}>\frac{-3 \alpha_{i} f_{i}}{h_{i}}
$$

$$
P_{i}^{\prime}(1)<\frac{3 P_{i}(1)}{h_{i}} \Rightarrow \frac{2 \beta_{i} f_{i+1}-2 \gamma_{i} f_{i+1}+\beta_{i} h_{i} d_{i+1_{i}}}{h_{i}}<\frac{3 \beta_{i} f_{i+1}}{h_{i}}
$$

Both conditions (7) and (8) can be further simplified to obtain the following:

$$
\gamma_{i}>-\alpha_{i}\left(\frac{h_{i} d_{i}}{2 f_{i}}+\frac{1}{2}\right)
$$

and similarly

$$
\gamma_{i}>\beta_{i}\left(\frac{h_{i} d_{i+1}}{2 f_{i+1}}-\frac{1}{2}\right)
$$

Combining Conditions (9) and (10), the rational cubic spline is positive i.e. $S_{i}(x)>0, \quad i=0,1,2, \ldots, n-1$ when the parameter $\gamma_{i}>0, i=0,1, \ldots, n-1$ satisfies

$$
\gamma_{i}>\operatorname{Max}\left\{0,-\alpha_{i}\left(\frac{h_{i} d_{i}}{2 f_{i}}+\frac{1}{2}\right), \beta_{i}\left(\frac{h_{i} d_{i+1}}{2 f_{i+1}}-1\right)-\frac{1}{2}\right\}
$$

Furthermore, this condition can be written as:

$$
\gamma_{i}=\chi_{\mathrm{i}}+\operatorname{Max}\left\{0,-\alpha_{i}\left(\frac{h_{i} d_{i}}{2 f_{i}}+\frac{1}{2}\right), \beta_{i}\left(\frac{h_{i} d_{i+1}}{2 f_{i+1}}-1\right)-\frac{1}{2}\right\}, i=0,1, \ldots, n-1
$$

such that $0<\chi_{i} \leq 0.50$. 


\section{Numerical results}

To test the positivity preserving by using the proposed rational cubic spline, we use the wind velocity data given in Table 1. Fig. 2 shows the examples of various interpolating curves produces by using cubic spline interpolations and the proposed scheme. Fig. 2(a) shows the interpolating curve when $\alpha_{i}=\beta_{i}=\gamma_{i}=1$ for all $i=0,1, \ldots, 6$. This is cubic Ball polynomial. Figs. 2(b) and 2(c) show the interpolating curve by using cubic spline interpolation with nota-knot and natural end conditions respectively. Clearly both cubic splines fail to preserves the positivity of the wind velocity data. Fig. 2(d) shows the example of interpolating curve by using PCHIP [1] that well documented in MATLAB software PCHIP can preserves the positivity of wind velocity, but the interpolating curve is not visually pleasing since on some intervals, the interpolating curves are very tight as well as very sharp. To avoid this, we implement the proposed scheme with positivity shape preserving. Figs. 2(f) until 2(h) show the example of positive interpolating curve by using the proposed scheme with various value of the free parameters $\alpha_{i}$ and $\beta_{i}$.

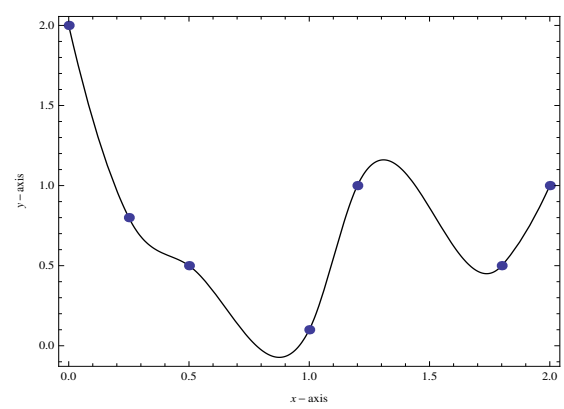

(a) The proposed scheme with $\alpha_{i}=\beta_{i}=\gamma_{i}=1$

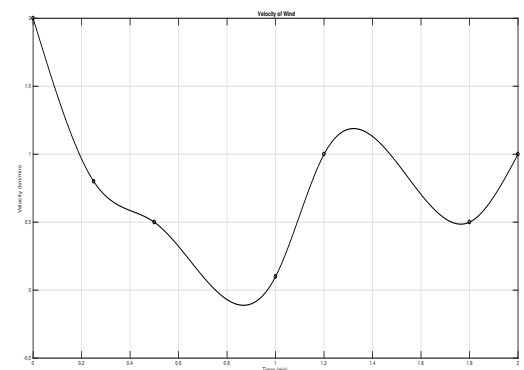

(c) Natural cubic spline

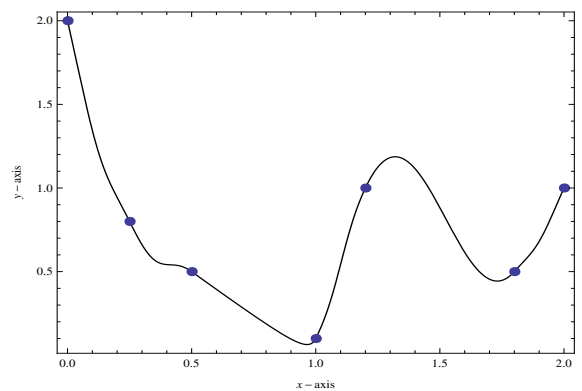

(e) The proposed scheme with $\alpha_{i}=\beta_{i}=1$

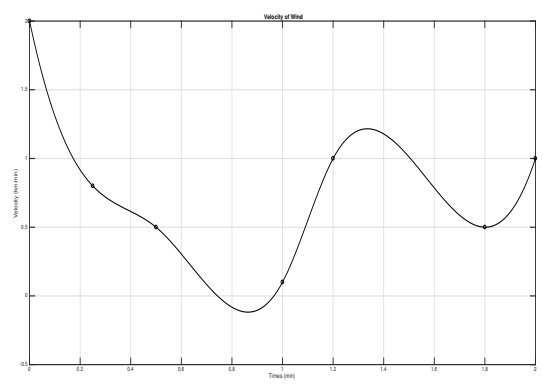

(b) Not-a-knot cubic spline

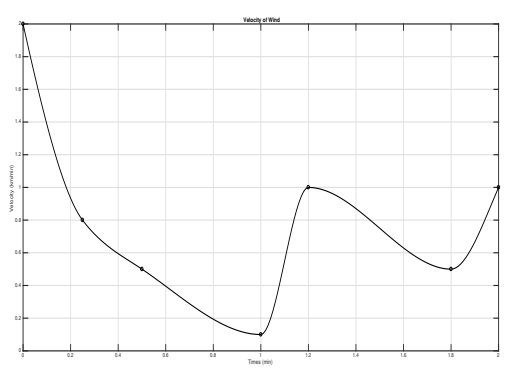

(d) PCHIP from MATLAB

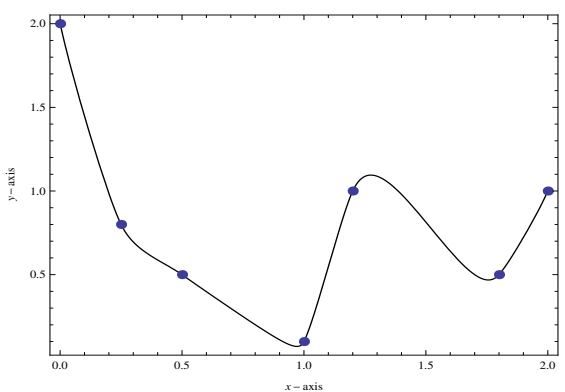

(f) The proposed scheme with $\alpha_{i}=\beta_{i}=0.25$ 


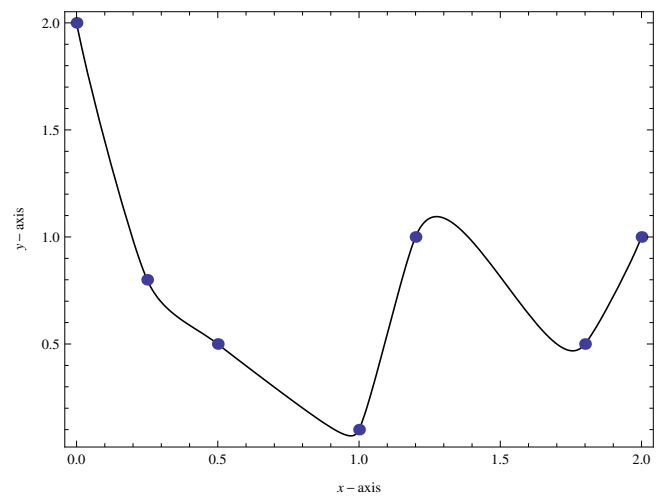

(g) The proposed scheme with $\alpha_{i}=\beta_{i}=0.1$

Fig. 2. Comparison of interpolating curve for wind velocity data.

\section{Discussion and conclusion}

The main objective of the present study is to preserves the positivity of wind velocity data by using new rational cubic spline interpolant. This rational spline has three parameters and the positivity of the data can be preserved by finding the suitable sufficient condition on one parameter meanwhile the remaining two free parameters can be used to refine the positive interpolating curve. Based on the comparison against (a) cubic spline interpolations with nota-knot and natural end boundary conditions, cubic Ball interpolation and PCHIP from [1], we conclude that, the proposed schemes better, notably in the aspects of visually pleasing and the existence of free parameters for shape refining as well as avoiding any derivative modification that has appeared in PCHIP. For future studies, we intend to extend the proposed scheme to surface interpolation. This is very useful for geological surface data interpolation.

Acknowledgement to Universiti Teknologi PETRONAS (UTP) for the financial support through research grant: YUTP: 0153AA-H24 including the MATLAB and Mathematica Software.

\section{References}

1. F.N., Fritsch and R.E. Carlson. SIAM J. Numer. Anal. 17: 238-246 (1980).

2. M.Z., Hussain, M. Sarfraz and T.S. Shaikh. Egyptian Informatics Journal 12: 231-236 (2011).

3. S.A.A., Karim and V.P. Kong. Research Journal of Applied Sciences, 9(4): 214-223 (2014).

4. S.A.A. Karim. Rational cubic spline interpolation for monotonic interpolating curve with $C^{2}$ continuity. MATEC Proceeding for SES 2017,UTP-UMP, Malaysia, Article No: 04016 (2017).

5. X. Qin, L. Qin and Q. Xu. IAENG International Journal of Computer Science. 43(2):2-11(2016).

6. M., Sarfraz, M.Z., Hussain and A. Nisar. Applied Mathematics Computation. 216:2036-2049 (2010).

7. J.W., Schmidt and W., Hess. BIT 28, 340-352 (1988).

8. J., Wu, X. Zhang and L. Peng. Mathematical Problems in Engineering. Article ID 964528, 10 pages (2010). 